\section{Gene expression signature of the ageing lung: breathing new life into COPD}

\author{
Steven Booth, Tillie-Louise Hackett
}

Due to consistently low birth rates and a higher life expectancy, countries within the European Union are transitioning to a much older population structure. ${ }^{1}$ The average number of older persons in the total population will increase significantly in the coming decade, as a large proportion of the post-war, baby-boom generation will reach retirement. Compared with generations prior, the 'baby boomers' were the most physically fit generation; however, they also grew up when smoking rates and exposure to secondhand smoke were at their peak. ${ }^{2}$

Tobacco use is a risk factor for six of the eight leading causes of death in the world including respiratory and cardiovascular diseases, stroke and cancer. ${ }^{2}$ Of these diseases, chronic obstructive pulmonary disease (COPD) is a major growing cause of morbidity and mortality ${ }^{3}$ with smoking and secondhand smoke exposure being the most important causative factors of the disease. ${ }^{3}$ In the European Union, the total direct costs of COPD account for 56\% (€38.6 billion) of the total healthcare budget. As there is growing evidence that up to $50 \%$ of smokers will eventually develop COPD, ${ }^{4-6}$ the ageing 'baby-boomer' population will therefore have a significant effect on future healthcare demands. ${ }^{7}$

COPD is characterised by irreversible chronic airflow limitation that is caused by emphysematous destruction of lung elastic tissue and obstruction in the small airways due to occlusion of their lumen by inflammatory mucus exudates, narrowing and obliteration. In COPD, the airflow limitation measured by reduced forced expiratory volume in $1 \mathrm{~s}$, progresses very slowly over time-potentially due to the high redundancy of small airway generations. ${ }^{89}$ Therefore, despite symptoms such as cough and phlegm being associated with susceptible smokers aged 20-44 years who will develop COPD, loss of lung function is

Department of Anesthesiology, Pharmacology and Therapeutics, Centre for Heart Lung Innovation, University of British Columbia, Vancouver, Canada

Correspondence to Dr Tillie-Louise Hackett, Department of Anesthesiology, Pharmacology and Therapeutics, Centre for Heart Lung Innovation, University of British Columbia, Vancouver, BC V6T 1Z4, Canada; tillie.hackett@hli.ubc.ca only observed later in life-early 60 s. $^{10} 11$ Certainly the incidence of COPD dramatically rises with age as newly diagnosed COPD increases from $2 \%$ in the population aged $\leq 45$ years to $12 \%$ in patients aged $\geq 65$ years. $^{12}$

Ageing has always been a subject of human fascination. Ageing, is defined as a time-dependent progressive loss of physiological integrity, resulting in impaired function and increased vulnerability to death. ${ }^{13}$ The human lung grows progressively until we are $\sim 25$ years of age. During 'normal' physiological ageing, healthy subjects will lose on average $25 \%$ of their lung function by the age of 75 years. This loss of lung function in the ageing lung is due to loss of elasticity, alveolar enlargement, low-grade inflammation but, in contrast to emphysema, no destruction of alveolar walls. ${ }^{14}$

Rather than ageing being programmed by selected genes, it is now thought that the stochastic interaction between genes, environment and intrinsic defects of the organism, plays an important role in the energy devoted to organ/cell maintenance and repair. ${ }^{15}$ Current hypotheses on the molecular mechanisms of ageing encompass telomere attrition, epigenetic alterations, oxidative stress, DNA damage, stem cell exhaustion, cellular senescence, mitochondrial dysfunction, defective protein turnover and many others that have been reviewed elsewhere. ${ }^{121316}$ The concept of accelerated ageing contributing to disease is not new or indeed unique to the lung as abnormal ageing of tissues has been implicated in the pathogenesis of many complex diseases reviewed elsewhere. ${ }^{13} 15$ The role of accelerated lung ageing is a relatively unexplored avenue that could help to understand how tobacco smoke may accelerate lung ageing in COPD and provide insights into potential new COPD therapeutic targets.

Moving towards the goal of a better understanding the molecular alterations that occur within the lung throughout the ageing process, the study by de Vries ${ }^{17}$ investigates genes underlying lung ageing in general and abnormal lung ageing in COPD. de Vries presents a strong signature for lung ageing across three cohorts of well-phenotyped lung tissue samples, identifying 3509 genes, which are differentially expressed with age. Specifically, by employing a linear mixed-effects model with a stringent false discovery rate (FDR) cut-off to $<0.0001$, they identify 1980 genes that increase expression with age, and 1529 genes that decrease expression with increasing age. Pathway analysis revealed that genes upregulated with age are enriched for biological processes related to calcium signalling and immune responses, whereas genes downregulated with increasing age were enriched for processes related to lung development and cell-cell contacts. Importantly, the age-related lung tissue gene expression signature identified by the authors demonstrates a significant overlap (33.5\%) with that from the Genotype-Tissue Expression Consortium (GTEx) study by Yang et al, which provided an extensive study of genes and pathways associated with ageing in multiple organ tissues. ${ }^{18}$ de Vries further proposes that ectodysplasin A2 receptor (EDA2R) could be a strong candidate gene and biomarker for lung ageing and, interestingly, the authors themselves note that EDA2R was the second highest ranked gene associated with lung ageing in the GTEx project. ${ }^{18}$ The EDA2R gene encodes a transmembrane receptor that belongs to the tumour necrosis factor receptor superfamily ${ }^{19}$ and has been shown to be transcriptionally regulated by $\mathrm{p} 53 .^{20}$ While the exact function of EDA2R and its role in lung ageing needs to be explored, the use of EDA2R as a biomarker specific to lung ageing may be limited as it was also shown in the aforementioned GTEx study by Yang et al to be highly associated with ageing of adipose, artery, heart, muscle and skin tissues. ${ }^{18}$

Moving on from the general concept of lung ageing, de Vries applied an interaction term in the linear mixed-effects model to identify genes that were differentially expressed with age between COPD and control tissue samples. This analysis yielded a set of 69 genes that were differentially expressed with age between COPD and control samples, using a more lenient FDR cut-off of $<0.25$. Gene set enrichment analysis of the ranked gene lists for COPD*age showed genes that were upregulated more with age in patients with COPD were enriched for the mechanistic target of rapamycin (mTOR) signalling pathway, splicing of introns and exons, and genes that belong to the ribosome complex, including the core-enriched genes FAU and RPL38. Conversely, genes that were downregulated with age more in patients with COPD were enriched in extracellular 
matrix (ECM)-receptor interaction pathways (including collagen genes COL6A3, COL3A1 and COL4A1). The implications of these results are as patients with COPD age they have a decrease in ECM-associated gene expression, which follows with the well-established pathophysiology of emphysema known to involve the degradation of ECM and progressive destruction of the alveolar walls. On the other side of this pathogenic seesaw, there is an increased expression of mTOR signalling pathways that could contribute to the abnormal cellular senescence, metabolism and growth seen in the COPD lung. ${ }^{16}$

The findings from de Vries are novel and indeed exciting as they provide evidence of a gene signature associated with accelerated ageing in COPD. However, the authors themselves note some important limitations of the study including differences in demographics of the three cohorts used with respect to their age, smoking status, lung function and severity of disease. Indeed the current study includes paediatric subjects (youngest 4 years of age) and thus it is not surprising that the authors show a number of lung development processes, which are downregulated with age. It would be of interest to see if these same genes and pathways are still identified after removal of the paediatric subjects. In the future, it would also be exciting to see if the age-related lung tissue gene-expression reported can actually predict accelerated ageing in COPD patients. In fact, such an approach was applied by Yang et al, whereby the authors used their derived gene signature associated with age to estimate/predict the tissue-specific apparent ages or relative ages in the population using both unsupervised (Principle Component Analysis) and supervised learning methods (Elastic Net Regression). However, this also highlights an important problem in the field of how we define accelerated ageing in lung diseases such as COPD verses chronological age. Indeed the recent approaches for the accurate correlation of gene expression signatures with careful assessment of tissue pathology in idiopathic pulmonary fibrosis ${ }^{21}$ and COPD $^{22}$ have enabled molecular characterisation of dominant pathogenic processes at different disease stages. Overall, current knowledge in the molecular mechanisms of lung ageing is very limited. Studies such as the one by $\mathrm{d} e$ Vries are important if we are to understand how molecular ageing mechanisms could be minimised to improve the quality of life of patients with obstructive lung diseases such as COPD.

Contributors SB and T-LH drafted, reviewed and approved the final version of the manuscript.

Funding This study was funded by Canadian Institutes of Health Research (LEKG).

Competing interests None declared.

Provenance and peer review Commissioned; externally peer reviewed.

(C) Article author(s) (or their employer(s) unless otherwise stated in the text of the article) 2018. All rights reserved. No commercial use is permitted unless otherwise expressly granted.

\section{D) Check for updates}

To cite Booth S, Hackett T-L. Thorax 2018;73:605-606.

Accepted 5 February 2018

Published Online First 15 February 2018

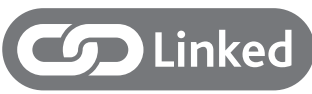

- http://dx.doi.org/10.1136/thoraxjnl-2017-210074

Thorax 2018;73:605-606.

doi:10.1136/thoraxjnl-2017-211173

\section{REFERENCES}

1 Eurostat. Population structure and ageing in the European Union (EU). 2017 http://ec.europa.eu/ eurostat/statistics-explained/index.php/Population structure_and_ageing

2 World Health Organization (WHO). R.O.f.E. Tobacco Data \& Statistics. http://www.euro.who.int/en/healthtopics/disease-prevention/tobacco/data-and-statistics (accessed Nov 2017).

3 Mannino DM, Buist AS. Global burden of COPD: risk factors, prevalence, and future trends. Lancet 2007;370:765-73.

4 Lundbäck B, Lindberg A, Lindström $M$, et al. Obstructive Lung Disease in Northern Sweden Studies. Not 15 but $50 \%$ of smokers develop COPD?--Report from the Obstructive Lung Disease in Northern Sweden Studies. Respir Med 2003;97:115-22.

5 Marsh S, Aldington S, Shirtcliffe P, et al. Smoking and COPD: What really are the risks? Eur Respir J 2006;28:883-4

6 Rennard SI, Vestbo J. COPD: the dangerous underestimate of 15\%. Lancet 2006;367:1216-9.

7 Pauwels RA, Buist AS, Calverley PM, et al. Global strategy for the diagnosis, management, and prevention of chronic obstructive pulmonary disease. NHLBI/WHO Global Initiative for Chronic Obstructive Lung Disease (GOLD) Workshop summary. Am I Respir Crit Care Med 2001;163:1256-76.

8 Hogg JC, Paré PD, Hackett TL. The contribution of small airway obstruction to the pathogenesis of chronic obstructive pulmonary disease. Physiol Rev 2017;97:529-52.

9 Mead J. The lung's "quiet zone". N Eng/ J Med 1970;282:1318-9.

10 Allinson JP, Hardy R, Donaldson GC, et al. The presence of chronic mucus hypersecretion across adult life in relation to chronic obstructive pulmonary disease development. Am J Respir Crit Care Med 2016;193:662-72.

11 de Marco R, Accordini S, Cerveri I, et al. Incidence of chronic obstructive pulmonary disease in a cohort of young adults according to the presence of chronic cough and phlegm. Am J Respir Crit Care Med 2007;175:32-9.

12 Meiners S, Eickelberg 0, Königshoff M. Hallmarks of the ageing lung. Eur Respir J 2015;45:807-27.

13 López-Otín C, Blasco MA, Partridge L, et al. The hallmarks of aging. Cell 2013;153:1194-217.

14 Fukuchi Y. The aging lung and chronic obstructive pulmonary disease: similarity and difference. Proc Am Thorac Soc 2009;6:570-2.

15 Kirkwood TB. Understanding the odd science of aging. Cell 2005;120:437-47.

16 Mercado N, Ito K, Barnes PJ. Accelerated ageing of the lung in COPD: new concepts. Thorax 2015;70:482-9.

17 de Vries M, Faiz A, Woldhuis RR, et al. Lung tissue gene-expression signature for the ageing lung in COPD. Thorax 2018;73:609-17.

18 Yang J, Huang T, Petralia F, et al. Synchronized agerelated gene expression changes across multiple tissues in human and the link to complex diseases. $\mathrm{Sci}$ Rep 2015;5:15145.

19 Yan M, Wang LC, Hymowitz SG, et al. Two-amino acid molecular switch in an epithelial morphogen that regulates binding to two distinct receptors. Science 2000;290:523-7.

20 Brosh R, Sarig R, Natan EB, et al. p53-dependent transcriptional regulation of EDA2R and its involvement in chemotherapy-induced hair loss. FEBS Lett 2010;584:2473-7.

21 Richards TJ, Kaminski N, Baribaud F, et al. Peripheral blood proteins predict mortality in idiopathic pulmonary fibrosis. Am J Respir Crit Care Med 2012;185:67-76.

22 Campbell JD, MCDonough JE, Zeskind JE, et al. A gene expression signature of emphysema-related lung destruction and its reversal by the tripeptide GHK. Genome Med 2012;4:67. 